\title{
Trigger and reconstruction for heavy long-lived charged particles with the ATLAS detector
}

\author{
S. Tarem ${ }^{1, a}$, S. Bressler ${ }^{1}$, H. Nomoto ${ }^{2}$, A. Di Mattia ${ }^{3}$ \\ ${ }^{1}$ Faculty of Physics, Technion, Israel Institute of Technology, Haifa 32000, Israel \\ ${ }^{2}$ Department of Physics, University of Tokyo, Tokyo 113-0033, Japan \\ ${ }^{3}$ Department of Physics, Michigan State University, East Lansing, MI 48824, USA
}

Received: 18 June 2008 / Revised: 2 March 2009 / Published online: 14 May 2009

(C) Springer-Verlag / Società Italiana di Fisica 2009

\begin{abstract}
Long lived charged particles are predicted by many models of physics beyond the standard model (SM). The common signature of such models is a heavy longlived charged particle with velocity smaller than the speed of light, $\beta<1$. This unique signature makes the search for it model independent. This paper presents methods we developed as part of the ATLAS trigger and reconstruction chain for identifying slow particles and measuring their mass. The efficacy of these methods is demonstrated using two models that are different in every aspect except for the existence of long lived charged particles; a GMSB model that includes sleptons with a mass of $100 \mathrm{GeV}$, and R-Hadrons with a mass of $300 \mathrm{GeV}$ produced in a split SUSY model.
\end{abstract}

\section{Introduction}

Long lived charged particles are allowed by many models of physics beyond the standard model (SM). In general, different models have different characteristics. However, long lived charged particles have a common characteristic in many models; when produced at the LHC some of them would travel with velocity significantly lower than the speed of light. Although the momentum (and therefore $\beta$ ) spectrum of those particles is model dependent, the common signal is a heavy long-lived charged particle with velocity smaller than the speed of light, $\beta<1$. This unique signature makes the search for it model independent.

ATLAS was designed to fully exploit the LHC discovery potential [1,2]. Nevertheless, the scenario of slowly moving particles was not considered. With $\beta<1$, a particle may be lost during data collection, or be marked as coming from the wrong event [3]. In this paper we consider the repercussions

a e-mail: shlomit.tarem@cern.ch of low particle speed in the ATLAS trigger and data acquisition design, and offer solutions to the problems encountered.

The new particles which have $\beta$ values close to unity are indistinguishable from ordinary muons. However, as we will demonstrate, particles with $\beta$ significantly lower than 1 can be identified and their mass measured. Such a search is model independent since the velocity and mass of the new particle are measured directly, without relying on other event features which are characteristic of a model. We demonstrate the model independence by showing efficiency and mass measurement results for two models that are different in every aspect except for the existence of long lived charged particles; Gauge Mediated SUSY Breaking point 5 (GMSB5), and R-Hadrons (hadronized gluinos) with a mass of $300 \mathrm{GeV}$.

This paper presents methods we developed in ATLAS for triggering on heavy long lived particles and measuring their mass. The separation between signal and background is based on the particle velocity and mass measurements, and does not include additional event characteristics. We show that a preliminary selection of heavy long lived particles can be made by the second level trigger, using the Resistive Plate Chambers (RPC) in the barrel of the muon spectrometer [4]. Our program to do this selection has already been incorporates in the second level trigger program. This has a negligible effect on the muon trigger rate but allows early detection of candidates, and by preventing rejection at later stages of the trigger, increases the robustness for the selection of heavy long lived particles.

Not all the data required to measure the heavy long lived particle are expected to be kept for the analysis stage. Specifically, the position and timing information of hits may not be available in the Analysis Object Data (AOD) which is the output of reconstruction and used for analysis. Therefore we developed tools for measuring $\beta$ at the reconstruction stage. We use one of the muon reconstruction programs to 
do this. The program adds the particle velocity to the AOD, allowing the selection of candidates for further study. The $\beta$ reconstruction is performed by refining the RPC measurement made in the second level trigger, and using the Monitored Drift Tubes (MDT) which give precise measurements of muon hits, and the Thin Gap Chambers (TGC) which are the trigger chambers in the end-cap region.

We will show that a specific trigger we developed for slow particles will select slow particles cleanly and efficiently, and the velocity measurement in reconstruction will identify the slow particles. With these techniques, the events containing long lived charged particles are selected by identifying those particles, independent from other model dependent event characteristics. If further analysis is required, the tagging of the candidate will allow us to select the more detailed data for a refined analysis.

\section{Motivation}

Generally speaking, a particle may become long lived either if a conservation law prevents it from decaying (in this case the particle is completely stable), or if its decay coupling is highly suppressed. Current cosmological measurements restrict the existence of beyond the SM stable charged particles, as summarized in [5].

The decay coupling may be suppressed due to small coupling constants, small phase space or if the interaction is communicated via extremely heavy particles. Depending on the model and on the point within the model parameter space, one of the above occasionally gives rise to the existence of long lived charged particles.

In the following, we will demonstrate how long lived charged particles are obtained in specific models within the well motivated Supersymmetry (SUSY) framework. Detailed description of models with long lived charged particles can be found in [5].

\subsection{Gauge mediated SUSY breaking (GMSB)}

The original motivation for the search for long lived charged particles arises from GMSB models [6]. In GMSB the symmetry breaking information is mediated by new chiral supermultiplets (the messenger fields) which couple to both the hidden and observable sectors. The coupling to the observable sector is through the ordinary $S U(3)_{C} \times S U(2)_{L} \times$ $U(1)_{Y}$ gauge bosons.

In GMSB the lightest SUSY particle (LSP) is the gravitino. If R-Parity is conserved, the next to lightest SUSY particle (NLSP) must decay to the gravitino. The NLSP decay rate is a free parameter in the model, but depends on the nature of the secluded sector. When the coupling is small, the NLSP may become long lived.
The GMSB particle spectrum is determined by a set of five parameters: $\Lambda$, the SUSY breaking scale, $M_{m}$, the messenger mass scale, $N_{m}$, the number of messenger fields, $\tan \beta$, the ratio of the vacuum expectation values of the two Higgs fields, sign of $\mu$, the higgsino mass term, $C_{\text {grav }}$, the scale factor of the gravitino mass, which determines the NLSP lifetime $\left(\tau_{N L S P} \sim C_{\text {grav }}^{2}\right)$. If $N_{m}>3$ the NLSP is often a slepton. The size of $C_{\text {grav }}$ determines its lifetime.

In GMSB the slepton is usually a product of a long decay chain starting from squarks or gluionos produced at the primary interaction. The events are expected to include many jets and other leptons.

\subsection{Split SUSY}

The term "split" in split SUSY refers to a big mass difference between the scalar masses (except the ordinary Higgs) which are very high, and the gaugino and higgsino masses which remain at the weak scale. Such a mass split may be a result of SUSY breaking at a high scale, which drives the scalar masses to high values whilst the gaugino masses are protected by a chiral symmetry [7].

In split SUSY the gluino can only decay through the exchange of virtual heavy squarks, and hence may become long lived. The gluino lifetime as a function of $m_{s}$, the SUSY breaking scale is given in [5]. Since the gluinos are colored particles, they will hadronize into color singlets, and the long lived particle to look for is, therefore, the R-Hadron.

In Split SUSY, the R-Hardons would be produced backto-back in the primary interaction, with few other energetic particles in the event.

\section{Heavy particle signature in ATLAS}

A long lived charged particle interacts in the detector in a way similar to a muon. Neither EM showers nor substantial hadronic showers are expected. Figure 1 shows the energy

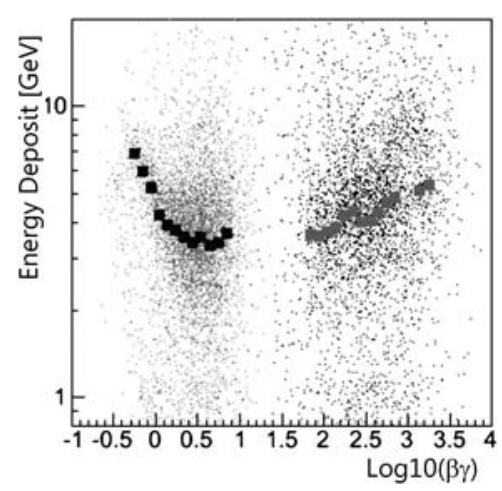

Fig. 1 Energy loss in the ATLAS calorimeter for simulated muons (black points on right) and sleptons (grey points on left) in GMSB5. The squares represent the mean energy loss in each bin 

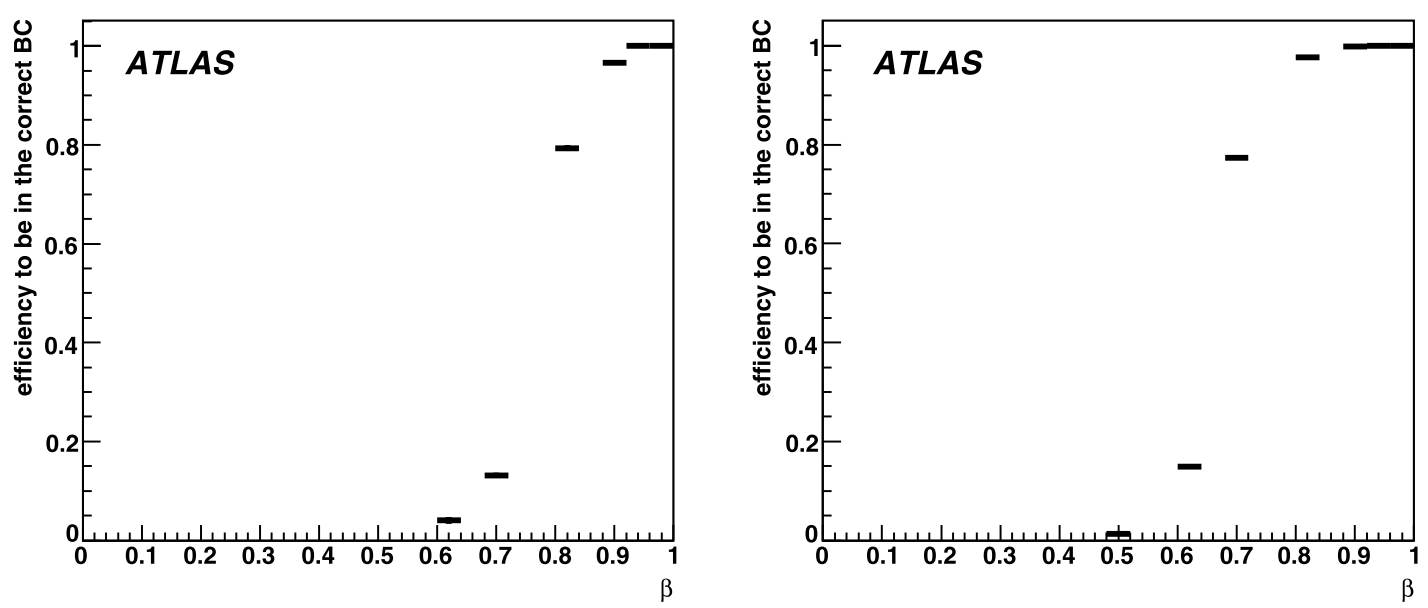

Fig. 2 The efficiency, as a function of $\beta$, for all slepton hits in the muon trigger chambers to be included in the same beam crossing (BC) with those from fast particles, for the endcap (left) and the barrel (right)

loss of simulated sleptons in GMSB5, one of the points chosen for ATLAS SUSY full simulation analysis, which will be discussed in more detail later. It can be seen that most sleptons have small energy loss in the calorimeter.

Energy loss is substantial only for very slow particles, which could be absorbed in the calorimeter if they have $\beta<0.3$. For these very slow particles, a different discovery method is required, which is not included in this work.

It has been shown [8] that the energy loss of R-Hadrons is also limited and they will also reach and pass the muon spectrometer. In R-parity conserving models, the gluino NLSP does not interact strongly with detector material, because the required squarks are too heavy. Even if R-Parity is violated, since the spatial extent of a wave function scales as $1 / M^{2}$, the cross section contribution of the heavy parton becomes negligible. The light quarks within the R-hadron interact strongly with detector material, but they hold only a very small fraction of the R-Hadron momentum since the gluino is so much heavier.

Thus the easiest and least model dependent signature to look for at ATLAS is a charged particle with low $\beta$ which reaches the muon chambers.

It is important to note that particles with $\beta \sim 1$ look like high $p_{\mathrm{T}}$ muons. Therefore, the presence of high $\beta$ long lived charged particles may result in significant excess of high $p_{\mathrm{T}}$ muons with respect to electrons and hence an apparent violation of lepton universality. This approach to the detection of long-lived particles does not allow a mass measurement and is outside the scope of this note.

When searching for slow particles at the LHC, it is essential to pay attention to the detector dimensions. Since the detector extends over $20 \mathrm{~m}$ in length from the interaction point to each detector side and the bunch crossing period is $25 \mathrm{~ns}$, this means that information from three separate bunch crossings co-exists in the detector at the same time.
To match correctly event fragments from different subdetectors, bunch crossing identification (BCID) is crucial [9]. This is calibrated so that particles originating together at the interaction point and traveling at the speed of light will have the same BCID assigned to them in all detector elements. When $\beta$ is sufficiently small, the particle will take longer to reach the detectors (especially those far from the interaction point) and hits may be assigned a wrong BCID and thus not be read-out. Figure 2 shows the efficiency of hits being all marked with the correct BCID in the muon trigger chambers. It can be seen that efficiency drops sharply below $\beta=0.8(0.7)$ at the endcap (barrel).

Nevertheless particles with $\beta$ between 0.5 and 0.7 can provide a trigger if the trigger time window is enlarged to values which were anticipated for calibration purposes. The effect of the enlarged trigger timing windows on trigger rate is expected to be similar to that of pileup and cavern background, which was shown to be small in [9]. The events could also easily be triggered by a different feature, an accompanying lepton or a large multiplicity of jets. Whatever the trigger, if the hits in the muon spectrometer are not acquired because of the late $\mathrm{BC}$, the particles and the opportunity to discover them are lost.

In order to find all hits from particles with $\beta<0.7$ in the barrel and $\beta<0.8$ in the endcap, ATLAS must collect hits from the following BC. Fortunately the MDT chambers collect data for $700 \mathrm{~ns}$, and thus hits from many BCs will be present. The RPC and TGC data are kept in a pipeline until the trigger decision is made and arrives back to the readout electronics. When the trigger signal arrives, the data acquisition can collect data from \pm 7 and \pm 1 BCs around the triggered $\mathrm{BC}$ respectively, without an additional trigger. However, the option to collect the extra BC, which was originally intended for debugging, may be switched on during routine ATLAS operation to improve efficiency for longlived charged particle. The expected background rate in the 
muon trigger chamber was simulated to amount to a few hits per readout sector per BC [9], so the effect of collecting muon trigger chamber hits from additional $\mathrm{BCs}$ on event size would be negligible.

\subsection{Background}

The background to the mass measurement of the long lived particle in the muon spectrometer is made almost exclusively of high $p_{\mathrm{T}}$ muons with mis-measured timing. In simulating the background we used $1000 \mathrm{pb}(200 \mathrm{pb})$ equivalent single muon events with $p_{\mathrm{T}}>40 \mathrm{GeV}\left(p_{\mathrm{T}}>100 \mathrm{GeV}\right)$ inside the ATLAS acceptance of $|\eta|<2.5$. This estimate follows approximately the cross section estimate of [10]. We used $200 \mathrm{pb}$ instead of the estimated $75 \mathrm{pb}$ of muon above $100 \mathrm{GeV}$ to take into account possible $p_{\mathrm{T}}$ dependence of the background to heavy particles.

The fake level for high $p_{\mathrm{T}}$ muons is quite low according to [9], and is covered by the increased muon cross-section we used. A small rate of background may also come from cosmic rays and new physics. These backgrounds are not treated in the note, but since the level-1 trigger rate from cosmic rays is estimated to be below $10 \mathrm{~Hz}$ for a $20 \mathrm{GeV}$ threshold, and the level-2 algorithm requires track pointing to the interaction point, we do not expected a big increase.

\section{Model related signature}

Although all heavy long-lived particles have $\beta<1$ in common, there are differences which arise from the event context and are model dependent. In particular, different models have different production cross sections and beta distributions. These characteristics affect the discovery potential. In the study presented below we demonstrate the ATLAS discovery methods using two different models: one in the framework of GMSB and one in the framework of split SUSY.

These two models are particularly interesting, not only because of their large production cross sections, but also because they differ in many aspects: the stable particle mass, $\beta$ spectra, production mechanism, SM interactions and accompanying features in the event. Thus, they may be considered as a test of model independence of the methods described.

\subsection{GMSB point 5 (GMSB5)}

Setting the GMSB parameters as follows: $\Lambda=30 \mathrm{TeV}$, $M_{m}=250 \mathrm{TeV}, N_{m}=3, \tan \beta=5, \operatorname{sign}(\mu)=+, C_{\text {grav }}=$ 5000 (ATLAS's GMSB5) results in a SUSY production cross section of $21 \mathrm{pb}$. The model spectrum has a gluino $(\tilde{g})$ mass of $705 \mathrm{GeV}$, squarks $(\tilde{q})$ with masses between 600 and $690 \mathrm{GeV}$ and neutralino $(\chi)$ masses between 113 and $335 \mathrm{GeV}$. The NLSP are long lived sleptons, with masses $M_{\tilde{\tau}}=102.2 \mathrm{GeV}$ and $M_{\tilde{e} / \tilde{\mu}}=100.3 \mathrm{GeV}$.

In this model, two sleptons are produced in the decay $\tilde{\chi}_{1}^{0} \rightarrow \tilde{l}+\bar{l}$, at the end of different cascade decays, hence they are produced with different $\beta$ 's. Figure 3 shows the $p_{\mathrm{T}}$ (left) and $\beta$ (right) spectra of the muons and NLSPs in GMSB5. As can be seen, the $\beta$ spectrum of the NLSPs covers a large range, with a tendency towards the high $\beta$ values because of the large mass difference between the squarks and gluinos that are produced in the hard collision, and the neutralinos and charginos that result from their decays. Due to the small mass difference between the neutralino and slepton, the slepton and anti-lepton are nearly collinear, and the events contain additional high $p_{\mathrm{T}}$ jets.

\subsection{Split SUSY—R-Hadron}

In split SUSY, two colored gluinos are produced at the primary interaction, so that both R-Hadrons are expected to be almost alone in the event, with a similar $p_{\mathrm{T}}$ and $\beta$. As can be seen in [11], the production cross section is high for low mass gluinos but decreases logarithmically with increasing mass. The $\beta$ spectrum tends to lower values with increasing mass.

It should be noted that R-Hadrons may change their charge in the calorimeter [12]. If the R-Hadron turns to neutral in the calorimeter, it will leave no trace in the muon
Fig. $3 p_{\mathrm{T}}$ (left) and $\beta$ (right) spectra of simulated muons and sleptons in GMSB5. Note that the muon are at $\beta=1$
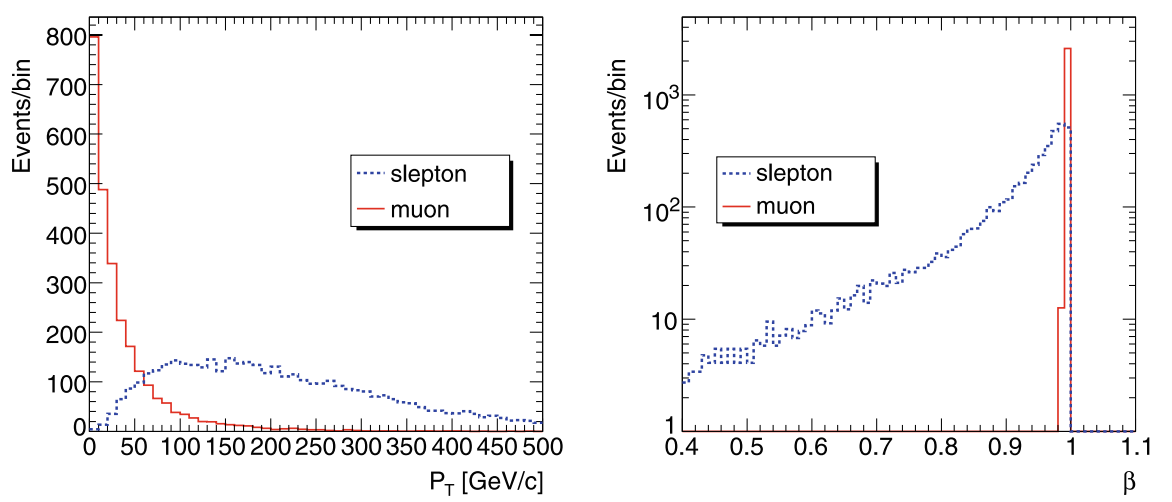
spectrometer and is lost. If the R-Hadron turns from neutral to charged in the calorimeter, it can be reconstructed in the muon spectrometer, but with no corresponding track in the inner detector. Such a situation is not treated correctly in the usual flow of trigger and reconstruction and requires special care.

\section{Simulated samples}

In order to stay as model independent as possible the tools presented below were developed using data sets of single particles. Using the generation program SingleParticleGun [13] we generated single staus of mass $m_{\tilde{\tau}}=100 \mathrm{GeV}$ with different velocities $(\beta=0.6,0.7,0.8,0.9,0.95)$ to study the potential signal behavior. The background is estimated using single muon files with the $p_{\text {T }}$ profile of inclusive muons.

The spectrum of GMSB5 was calculated using ISAJET V7.74 [14], and the R-Hadron events were produced using PYTHIA 6.403 [15]. For detailed description of the generation parameters see [11].

ATLAS's response to the events above was simulated using GEANT4 [16]. The characteristics of the long lived charged particle affect its interaction with the detector. Therefore, special patches (G4Extentions/RHadrons, Sleptons) within the ATLAS implementation of GEANT4 were used in order to simulate the passage of long lived sleptons and long lived R-Hadrons through the ATLAS detector. In both packages, the new stable particle masses are defined at the simulation step, so as to enable simulation with various masses. The slepton simulation defines the ionization energy loss, whereas the R-Hadron simulation must incorporate their hadronic interaction and charge flip probabilities as well [8].

$\mathrm{R}$-Hadrons have strong interactions with the detector material, but the energy loss of R-Hadrons in nuclear interaction is only due to the interactions of the light partons, and is small because the fraction of R-Hadron energy carried by the light partons is small. In the GEANT4 implementation the authors [8] use a purely geometric cross section, and phase space arguments are used to predict the different 2-to-2 and 2-to-3 reactions. The heavy parton is neglected because the spatial extent of a wave function scales as $1 / M^{2}$, making the cross section contribution negligible, so the heavy parton is just a reservoir of kinetic energy.

The total energy loss of R-Hadrons with a mass of $300 \mathrm{GeV}$ in 1 meter of iron is estimated to be between 5 and $11 \mathrm{GeV}$, depending on their kinetic energy. This will allow most R-Hadron to reach the muon spectrometer with most of their initial momentum, but the spread of energy loss is larger than in the case of sleptons.

\section{Triggering on long lived heavy charged particles}

ATLAS has a trigger system [2] to reduce the data taking rate from $40 \mathrm{MHz}$ to $\sim 100 \mathrm{~Hz}$, designed to keep the events that are potentially the most interesting. The first level trigger [9] selection is done by custom hardware and identifies a detector region and a beam crossing for which a trigger element was found. The second level trigger [17] is performed by dedicated software, making its decision based on data acquired from the BC and Region of Interest (RoI) found at level-1. Eventually, the event filter [17] uses the complete event data, using algorithms adapted from the offline reconstruction, to refine the selection of level-2 and add more complex signatures to the final selection. An event must pass all trigger levels in order to be kept for analysis.

\subsection{Level-1}

Level-1 triggers are produced by the calorimeter or the muon trigger chambers. Although different models may result in somewhat different trigger scenarios, a long lived charged particle is most likely to trigger as a muon. We provide a brief description of the features of the muon trigger relevant for a slow particle search.

The level-1 muon trigger is based on dedicated fast detectors: the RPCs in the barrel and the TGCs in the end-caps. The basic principle of the algorithm is to require a coincidence of hits in the different trigger stations within a road from the interaction point through the detector. The width of the road is related to the bending of the muon in the magnetic field and thus to the $p_{\mathrm{T}}$ threshold to be applied.

The level-1 muon trigger also identifies the beam crossing in which the collision occurred, so that all sub-detectors collect the data from it. The trigger decision itself may include hits from the adjacent $\mathrm{BC}$, depending on the programmed timing gate in the electronics.

The trigger in both the barrel and the end-cap regions is based on three trigger stations. The low- $p_{\mathrm{T}}$ triggers (4 to $10 \mathrm{GeV}$ ) arrive from a coincidence in two stations, while the high- $p_{\mathrm{T}}$ triggers (over $10 \mathrm{GeV}$ ) require an additional coincidence with the third station. In the barrel the high- $p_{\mathrm{T}}$ station is in the outer station of the muon spectrometer, approximately $10 \mathrm{~m}$ from the interaction point. In the end-cap all three trigger stations are in the middle of the muon spectrometer, between 13 and $15 \mathrm{~m}$ from the interaction point.

The hits from a slow particle may fall outside the correct $\mathrm{BC}$, either for all trigger stations (e.g. if the trigger was produced by another feature in the event) or hits in the low- $p_{\mathrm{T}}$ stations in the barrel may arrive at the correct $\mathrm{BC}$, but the hits in the outer station may be late. In such a case, even if the $p_{\mathrm{T}}$ of the particle was high, it would produce a low $p_{\mathrm{T}}$ trigger.

In GMSB5 the two sleptons are produced with different $\beta$ 's. A muon trigger in the correct $\mathrm{BC}$ is very likely, either 
Table 1 Level-1 triggers for GMSB5 events. The trigger type labels are used in the menu definitions

\begin{tabular}{lll}
\hline Trigger type & Description & Trigger efficiency \\
\hline$\mu 40$ & Muon with $p_{\mathrm{T}}>40 \mathrm{GeV}$ & $95 \%$ \\
$x E 200$ & Missing $E_{T}>200 \mathrm{GeV}$ & $63 \%$ \\
Em25i & Isolated EM cluster $E>25 \mathrm{GeV}$ & $46 \%$ \\
\hline
\end{tabular}

from a slepton with high $\beta$, or from one of the accompanying leptons. Nevertheless, a low $\beta$ slepton, one with good potential for a mass measurement, could arrive to the muon spectrometer, or more likely the outer muon station, in the next BC. In such a case the slow slepton will not be found by the trigger, or be identified as a low $p_{\mathrm{T}}$ muon. Table 1 summarizes the Level-1 trigger efficiencies for the GMSB5 sample, based on the Level-1 thresholds defined in the ATLAS trigger menu foreseen to be applied for a luminosity of $10^{33}$.

In Split SUSY, the gluinos are produced directly, and there are few other features in the event. Therefore the RHadrons themselves must trigger the event. Since both of the R-Hadrons may be slow, the muon trigger may be on the wrong BC. As a result, all other event information such as that from the inner detector may be lost in the previous BC. This problem may be solved by also collecting data from the previous $\mathrm{BC}$ in the inner detector, but the feasibility of doing this, from the point of view of increased data volume, has not been investigated in this work. Additional data from the calorimeter is not required in order to find long lived heavy charged particles.

Even if events containing new long-lived particles will pass the level-1 trigger, the data from a slower particle may arrive in the next $\mathrm{BC}$ and be lost, unless muon data from the next $\mathrm{BC}$ is also collected.

\subsection{A level-2 trigger for heavy sleptons}

At level-2, the algorithms are called based on the trigger elements found at level-1. Each algorithm has a reconstruction stage which processes the data from the relevant parts of the subdetectors, and a hypothesis stage which makes the decision to keep or reject the reconstructed feature. Level-2 has an average time allocation of $10 \mathrm{~ms}$ (on an $8 \mathrm{GHz} \mathrm{PC}$ ) to make the decision.

The level-2 suite for muons includes two stages. The first stage starts from a level-1 muon Region of Interest (RoI) and reconstructs the muon in the spectrometer, giving a new $p_{\mathrm{T}}$ estimate for the muon candidate and creating a new trigger element [18]. The hypothesis cuts on the estimated $p_{\mathrm{T}}$ so that $90 \%$ of the muons with $p_{\mathrm{T}}$ above the nominal threshold would pass the selection. The resulting trigger element then passes to the next algorithm, which matches an inner detector track with the trigger element from the muon spectrometer and refines the $p_{\mathrm{T}}$ estimate [19].

In the barrel region, a high $p_{\mathrm{T}}$ slow particle may trigger at level-1 only the highest low $p_{\mathrm{T}}$ threshold (using a two station coincidence) because it did not arrive in time to the outer muon station. In such a case the level-2 trigger will re-estimate the $p_{\mathrm{T}}$ using the MDT hits in the outer station, but only if the event is accepted at level- 1 . The level- $2 p_{\mathrm{T}}$ estimate using the stand-alone muon spectrometer algorithm may be poorer in such a case. This could be recovered by the matching with a track in the ID, in the cases where such a track exists.

We developed an algorithm to trigger on heavy stable charged particles, by measuring their velocity at the level-2 trigger. In the barrel of the muon spectrometer, the excellent time resolution (3ns) of the RPC chambers allows measurements of the time of flight (TOF). When a charged particle passes through an RPC chamber two signals are generated; one measuring its time and position in the phi direction and the other measuring it time and position in the eta direction. The time measurement includes the time of flight and in addition the propagation time from the point where the particle passed in the detector to the readout electronics.

The muon level-2 algorithm combines the separate hits in the two directions into one point in space, with $\eta$ and $\phi$ coordinates. Since both coordinates are known, the signal propagation time along RPC strips to the readout may be calculated and subtracted from the measured signal time, to give the signal production time which corresponds to the particle time of flight. Finally, the particle velocity is calculated from the average velocity of the hits on the track.

Measuring $\beta$ in the muon spectrometer at the second level trigger could be particularly useful for R-Hadrons. The second stage of the level-2 trigger requires matching between the candidate found in the muon spectrometer and a track in the inner detector. If the R-Hadron was neutral in the inner detector, or the hits in the inner detector were in the previous $\mathrm{BC}$, it will fail this test. The resulting efficiency for R-Hadrons with a mass of $300 \mathrm{GeV}$ was found to be about $50 \%$. However, if the R-Hadron is identified in the muon spectrometer as a slow particle candidate, it could be accepted without the requirement of a matching inner detector track. The corresponding muon rate is expected to be completely negligible since all muons have an inner detector track. Cosmic ray muons are only selected at level-2 if they project back to the interaction point, and thus will also have associated inner detector tracks.

Our method for measuring the particle speed at the level2 trigger has been included as a part of the first feature extraction algorithm. In the barrel, a slow particle may already be distinguished and selected effectively, at the level-2 trigger. Figure 4 shows the $\beta$ reconstructed at level-2 for sleptons of different $\beta$ and for muons. The $\beta$ resolutions are 
around $3 \%$ and increase to $3.5 \%$ for $\beta$ of 0.95 and $3.7 \%$ for muons. At low $\beta$ there is a downward bias of $\sim 5 \%$ mainly due to energy loss in the calorimeter.

A selection on the beta measurement by itself would leave too many muons in the sample, as can be seen in the left plot of Fig. 5. At the hypothesis stage, we select using $p_{\mathrm{T}}$ (candidate) $>40 \mathrm{GeV}, \beta$ (candidate) $<0.97$ and $m$ (candidate) $>40 \mathrm{GeV}$. The efficiency of this selection is 91\% for GMSB5 events with sleptons in the barrel, and 92\% (93\%) for R-Hadrons with a mass of $300 \mathrm{GeV}(1000 \mathrm{GeV})$ in the barrel. This corresponds to $58 \%$ of events passing the level-1 trigger over the full detector acceptance.

Figure 5 shows the mass distribution of GMSB5 signal and background resulting from the selection for an integrated luminosity of $500 \mathrm{pb}^{-1}$. It can be seen that for GMSB5 the signal to background ratio is already good at

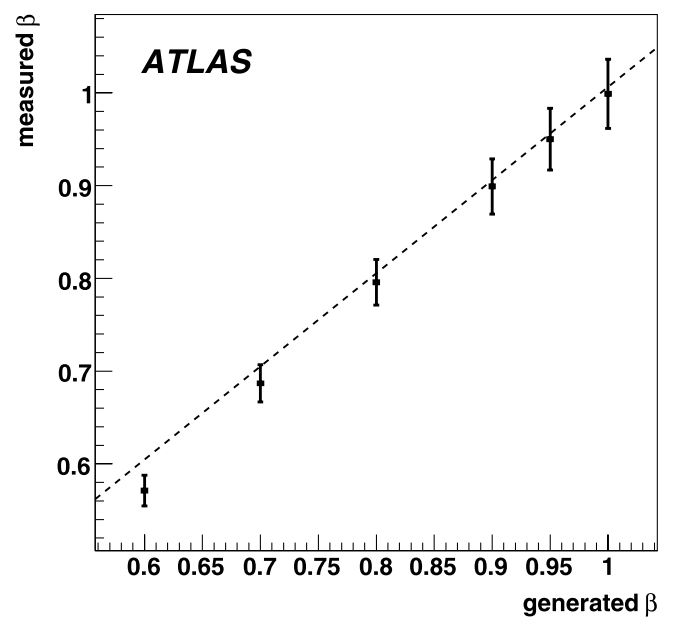

Fig. 4 The $\beta$ measured for sleptons in the barrel at level-2 for different values of true $\beta$. The error bar represents the fitted sigma of the measured $\beta$ distribution

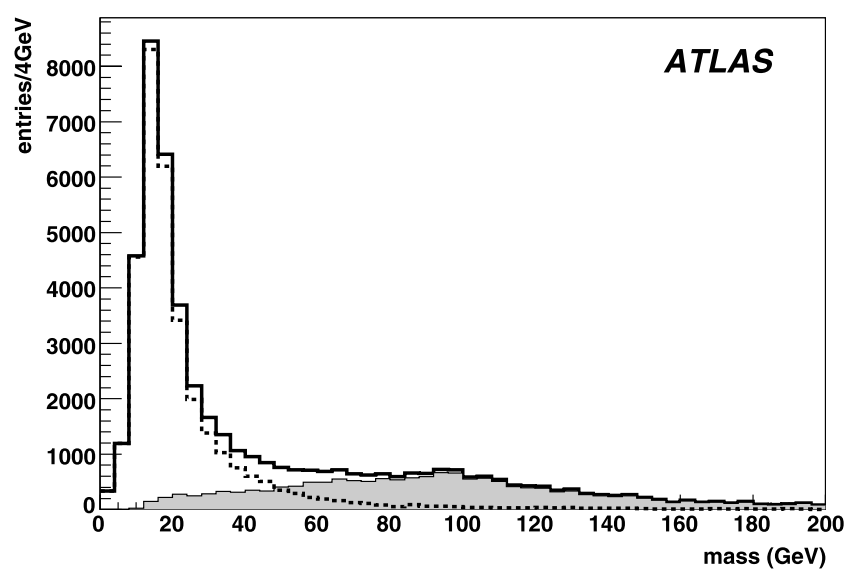

Fig. 5 Mass distribution of signal and background resulting from the level-2 selection for an integrated luminosity of $500 \mathrm{pb}-1$. On the left is the full mass range and on the right is the range accepted by the cuts. level-2. Figure 6 shows on the left the mass distribution for $100 \mathrm{pb}^{-1}$, and on the right the distribution for $500 \mathrm{pb}^{-1}$ when the slepton cross-section is reduced by a factor of 2 . The large width of the slepton mass distribution results from the inaccurate $p_{\mathrm{T}}$ estimate at the first stage of the level2 trigger. The $p_{\mathrm{T}}$ resolution can be improved by using a matching inner detector track, if it exists.

If there is no inner detector track, as in the case of an initially neutral R-Hadron, the resulting mass is coarsely measured, but sufficiently separated from the background. The slow particle selection at level-2 results in an efficiency of $92 \%$ to select events with R-Hadrons in the barrel. This compares favorably with the $50 \%$ efficiency of the muon trigger mentioned above. The muon rate for this selection is completely negligible since all muons have an inner detector track.

Our conclusion is that a slepton with the production characteristics (mass, $\beta$ distribution and cross section) of the GMSB5 point could be discovered using the tag from our level-2 trigger with an integrated luminosity as low as a few $\mathrm{pb}^{-1}$. Although the trigger was not designed for discovery, if physics beyond the standard model can be identified in the trigger, then this tagging will be used to enable an efficient selection of the samples to be further reconstructed and analyzed with offline software tools.

Our measurement of $\beta$ for high $p_{\mathrm{T}}$ muon candidates has already been incorporated in the standard ATLAS level2 program muFast [18], and a so-called $\tilde{\tau}$ hypothesis was added to make the selection described above.

Less than $2.4 \%$ of muons passing the $40 \mathrm{GeV}$ threshold in the barrel at the first stage of the level-2 trigger would also pass the stau hypothesis. Therefore, at a luminosity of $10^{33} \mathrm{~cm}^{-2} \mathrm{~s}^{-1}$, the slow particle trigger chain would produce a rate lower than $1 \mathrm{~Hz}$ coming from prompt muons. Additional interactions in the same $\mathrm{BC}$ and muons from cosmic



The shaded area is the signal, the dashed line is the muon background, and the full line is the sum 


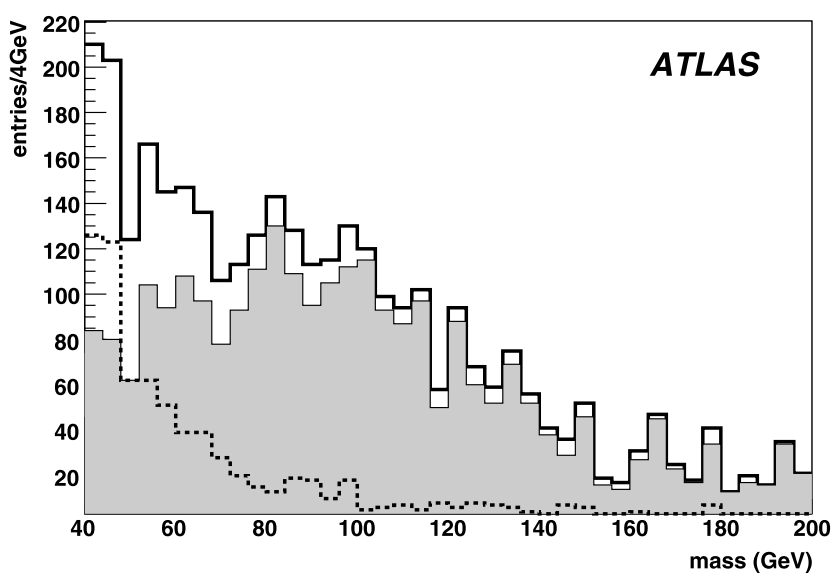

Fig. 6 Mass distribution of signal and background resulting from the level-2 selection for an integrated luminosity of $100 \mathrm{pb}-1$ (left), and with integrated luminosity of 500 pb-1 but the GMSB cross section

rays have only been studied with small samples, but these indicate that the total trigger rate will by less than $2 \mathrm{~Hz}$. Further refinement of the slow particle selection using subsequent muon trigger stages will reduce this rate to $0.2 \mathrm{~Hz}$. This rate fully overlaps with the muon trigger rate.

The limitations of the level-2 slow particle trigger should be noted: First, this selection is performed only in the barrel of the muon spectrometer, where the RPCs are the trigger chambers. The timing information from the endcap is in BC granularity and cannot be used to measure $\beta$. Second, a slow particle which does not produce a RoI in the correct BC will not cause the muFast algorithm to be called, and the selection will not be performed. Therefore, slow particles in the endcap, as well as very slow particles in events triggered by other fast particles in the event, can only be identified offline.

\subsection{Slow particles in the event filter}

The final trigger decision in ATLAS is made in the event filter, which uses algorithms adapted from the offline reconstruction. As will be shown in the next section, the standard muon reconstruction is not efficient for slow particles; therefore they would be rejected at this stage. The combined trigger efficiency of level-2 and the event filter for sleptons with velocity $\beta=0.6$ is $39 \%$.

Below we present a specific reconstruction algorithm for slow particles, which will avoid this efficiency loss. This algorithm is currently being adapted for the event filter.

Slow particle candidates found at level-2 that do not have a matching inner-detector track (such as charge flipped RHadron candidates) should be accepted without further event filter selection. This will increase the combined trigger efficiency of the level-2 and the event filter for R-Hadrons with a mass of $300 \mathrm{GeV}$ from $25 \%$ to $92 \%$ in the barrel. The muon rate for this selection is completely negligible since

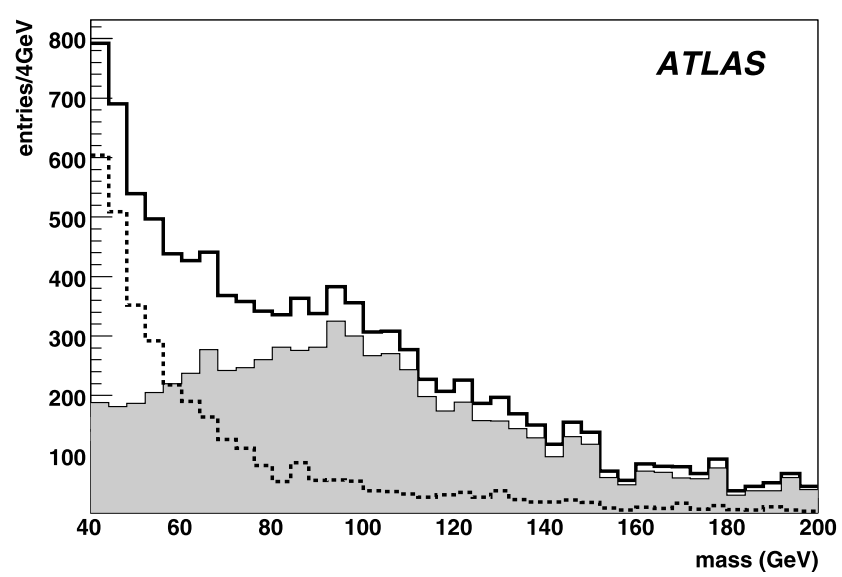

lower by a factor of 2 (right). The shaded area is the signal, the dashed line is the muon background, and the full line is the sum

all muons have an inner-detector track, but the effect of cavern background on this selection has not been studied yet.

\section{Reconstruction of slow particles}

Most new particle searches look for heavy particles which decay into known stable particles. The discovery in those cases is made by kinematic analysis on those known particles, which are all reconstructed by the ATLAS reconstruction packages. The search for a new stable charged particle is unique in that these particles require a custom reconstruction. Without a specific reconstruction, some of the new particles would be misidentified as muons, while many will be lost completely. Thus the identification of slow particles and the measurement of their velocity must be done at the reconstruction stage, and the measured velocity included in the resulting analysis object data.

\subsection{Reconstruction with the standard muon reconstruction packages}

In the standard ATLAS reconstruction, long lived charged particles are expected to be reconstructed as muons. Muons are reconstructed by forming track segments in the three stations of the Muon Spectrometer. Segments in the $\phi$ direction are found using the trigger chamber data.

The efficiency to reconstruct slow particles is compromised due to the following issues: the data may not be collected if the hits from a particle are in the next bunch crossing instead of the nominal BC of the event; the lack of a $\phi$ segment hinders the reconstruction of the precision segment in eta using the MDT data; the reconstructed radii of MDT hits are enlarged by late arrival of the slepton and may not fit well to a segment. 
The efficiency of reconstructing the slepton as a muon depends on the reconstruction program. Figure 7 shows the reconstruction efficiency for sleptons from GMSB5 with two ATLAS combined muon reconstruction packages [20] [21]. It can be seen that reconstruction efficiency starts dropping sharply for $\beta<0.75$. This indicates that special reconstruction will be required for the slow particles, similar to the one we describe below.

\subsection{Identifying heavy long lived particles at reconstruction}

Another approach is to estimate the slow particle mass in the event reconstruction. We do this with another ATLAS muon identification package [22], which starts from inner detector tracks and looks for corresponding hits in the muon spectrometer. This enables us to select candidates when the segment reconstruction is imperfect. In order to minimize the processing time, the velocity estimation is activated in the program when the candidate has $p_{\mathrm{T}}>20 \mathrm{GeV}$ and at least a few muon hits associated with the inner detector track.

The identification of heavy charged particles is based on three techniques.

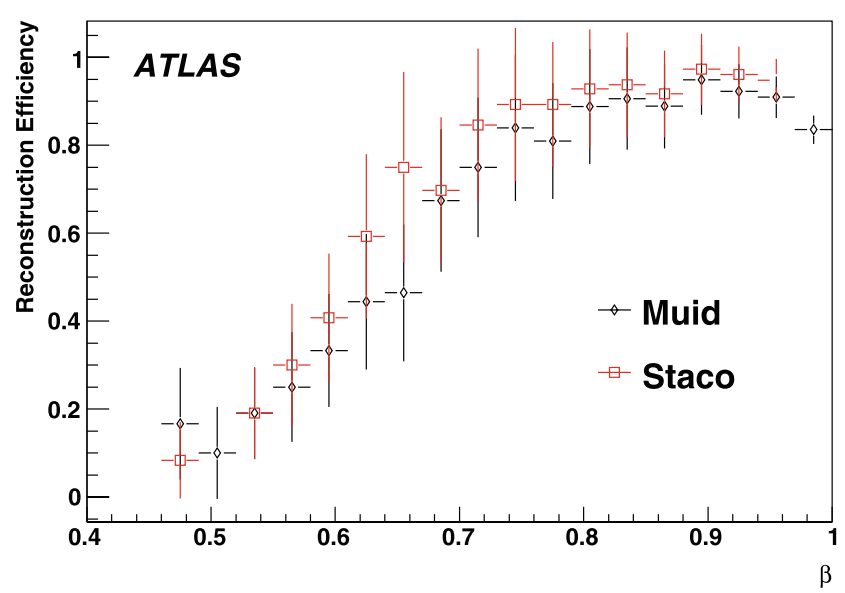

Fig. 7 Efficiency as a function of $\beta$ for two ATLAS combined muon reconstruction packages
- Recovering trigger detector hits from the next beam crossing.

- Estimating the particle velocity from the hit time in the RPC.

- Estimating the particle velocity by selecting $\beta$ that minimizes the $\chi^{2}$ of the MDT segments.

The lost efficiency in the trigger chambers may be recovered if we also include hits from the following BC. Figure 8 compares the number of TGC segments found for sleptons with $\beta=0.6$ when hits from the next $\mathrm{BC}$ are excluded (left) or included (right) in the reconstruction. For sleptons with $\beta=0.6$ using the hits from more TGC BCs improves the reconstruction efficiency in the end-cap from $65 \%$ to $97 \%$.

Offline, the particle velocity can also be measured using the MDTs, the ATLAS precision muon chambers. The MDT chambers are composed of layers of grounded tubes filled with gas. In the middle of each tube there is a wire to which a high voltage is applied. When a charged particle passes through a tube it ionizes the gas, and the ionized electron drifts toward the wire.

The hit position is obtained from the particle drift time, using a known relation between the drift distance and the drift time, $R=R\left(t_{\text {drift }}\right)$. The result is a ring of radius $R$ around the wire. A segment is reconstructed as a line tangent to the radii in the different layers as shown on the left side of Fig. 9.

The drift time is estimated by subtracting the expected time of flight, $t_{0}$, from the measured signal time. The expected $t_{0}$ is based on particles passing the detector at the speed of light. Slow particles have a longer time of flight, and therefore, when this is not taken into account, the measured radius is larger than the true one. Larger radii may result in either badly fitted segment or wrong direction of the segment as shown on the right side of Fig. 9.

Following the idea suggested by [23, 24], a minimization of the $\chi^{2}$ of the segment fit with respect to the time of arrival to the muon detectors is performed for each candidate, and for each group of MDT hits associated to an inner de-
Fig. 8 The number of TGC segments found for sleptons with $\beta=0.6$, using hits from the $\mathrm{BC}$ of the event (left) and using also hits from the next BC (right)
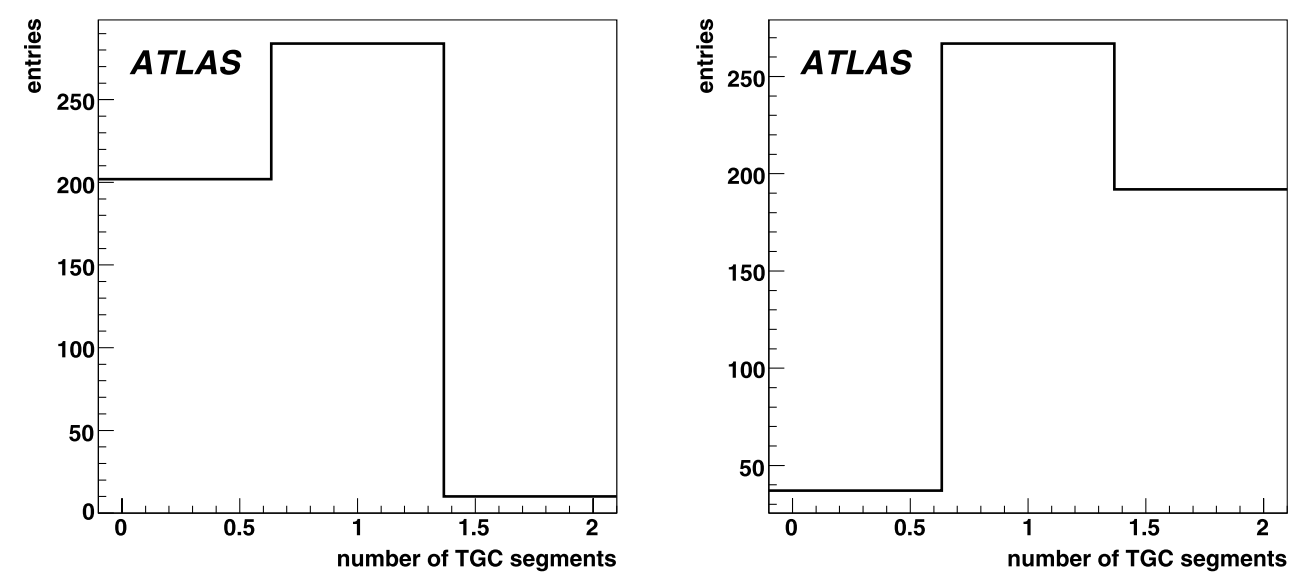
Fig. 9 A correct (left) and incorrect (right) segment reconstruction in an MDT chamber. The wrong reconstruction is due to longer time of flight which results in overestimation of the drift distance
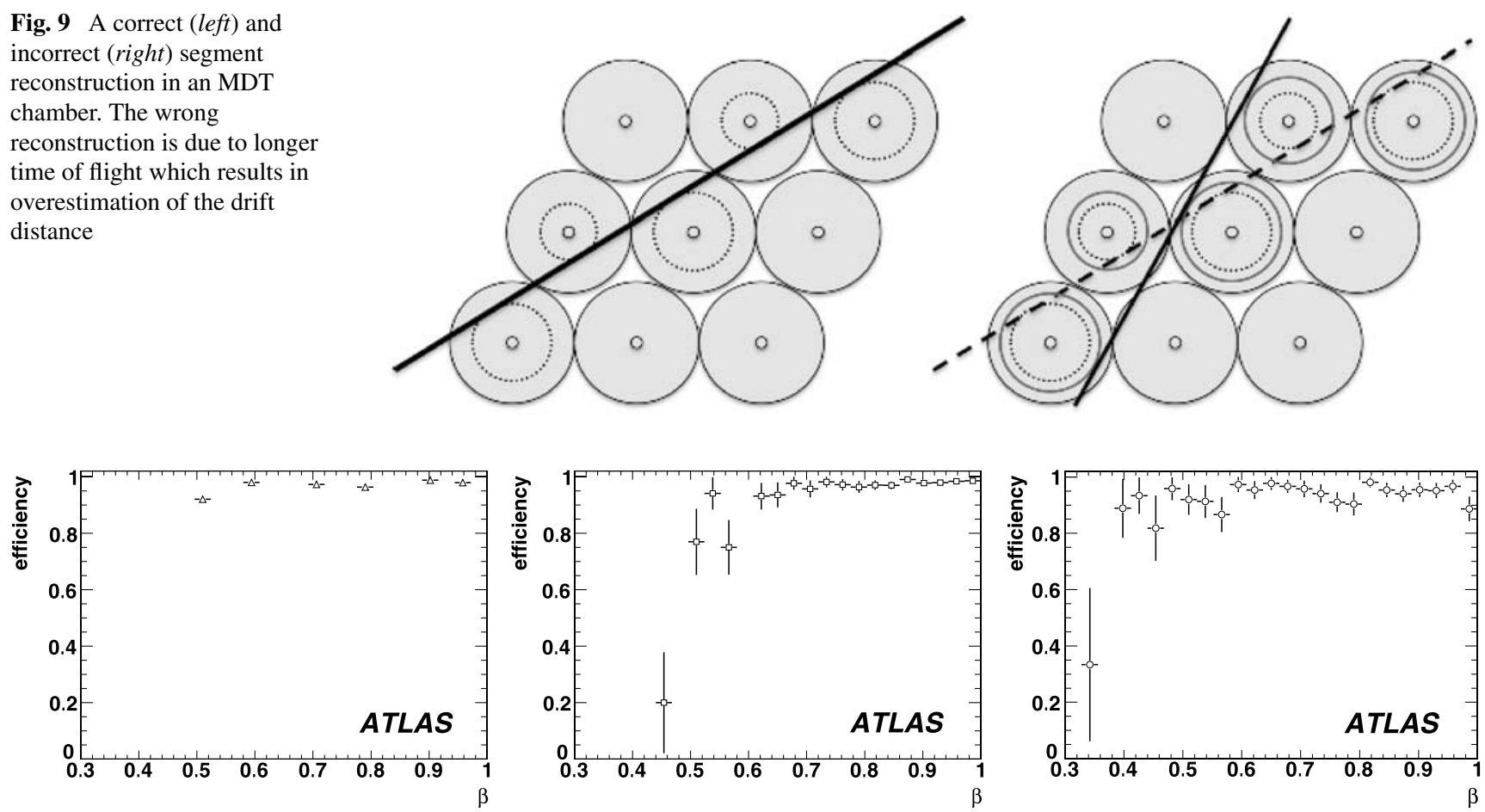

Fig. 10 Reconstruction efficiency for sleptons in single stau events (left), sleptons from GMSB5 (middle) and R-Hadrons (right) as a function of $\beta$ with a reconstruction program that also estimates $\beta$

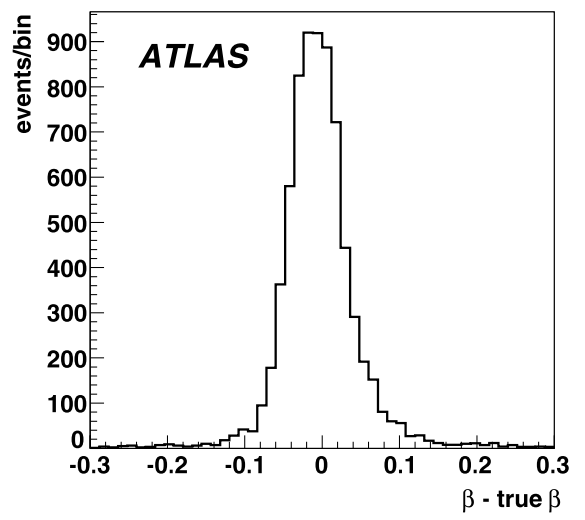

Fig. 11 The $\beta$ resolution of sleptons in single stau events (left) is centered at -0.006 with a $\sigma$ of 0.035 , for sleptons from GMSB5 (middle) the resolution is centered at -0.015 with a $\sigma$ of 0.030 . The $\beta$ res-
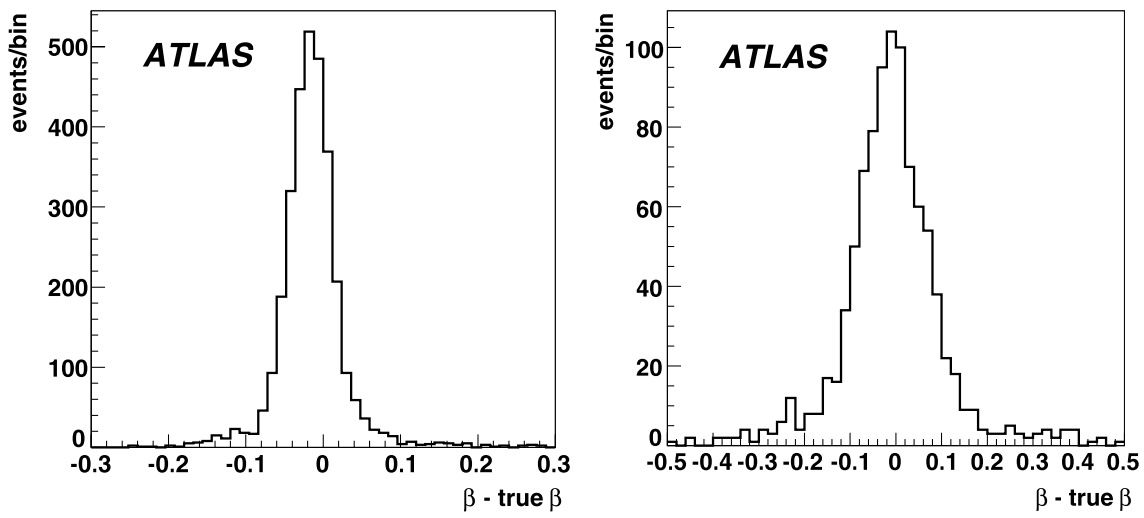

tector track. The algorithm yields an estimate of $\beta$ and of the particle mass per candidate.

An estimate of $\beta$ from the RPC muon trigger chambers, is performed in a similar way to the algorithm from the level-2 trigger. The estimates from the MDT and the RPC are combined to improve the $\beta$ estimate.

Refitting MDT hits with a $\beta$ hypothesis also increases the number of good segments found, and therefore the reconstruction efficiency. Figure 10 shows the efficiency of reconstructing sleptons and R-Hadrons as a function of $\beta$. Figure 10 Shows the efficiency for single stau generated with olution of R-Hadrons with a mass of $300 \mathrm{GeV}$ (right) is centered at -0.009 with a $\sigma$ of 0.070

specific $\beta$ values (left), GMSB5 sleptons (middle), and of R-Hadrons (right). All samples show reasonable efficiency measurement at low $\beta$. This may be compared with Fig. 7 which shows the reconstruction efficiency for sleptons with a standard muon reconstruction.

Figures 7 and 10 both present the efficiency, over the barrel and end-cap together, of combined reconstruction in the inner and muon detector for slow particles that had a track in the inner detector.

Figure 11 shows the $\beta$ resolution for sleptons in single stau events (left) sleptons from GMSB5 (middle) and R- 
Fig. 12 The efficiency to reconstruct a $\beta$ within \pm 0.1 from the true value for sleptons (left) and R-Hadrons (right)
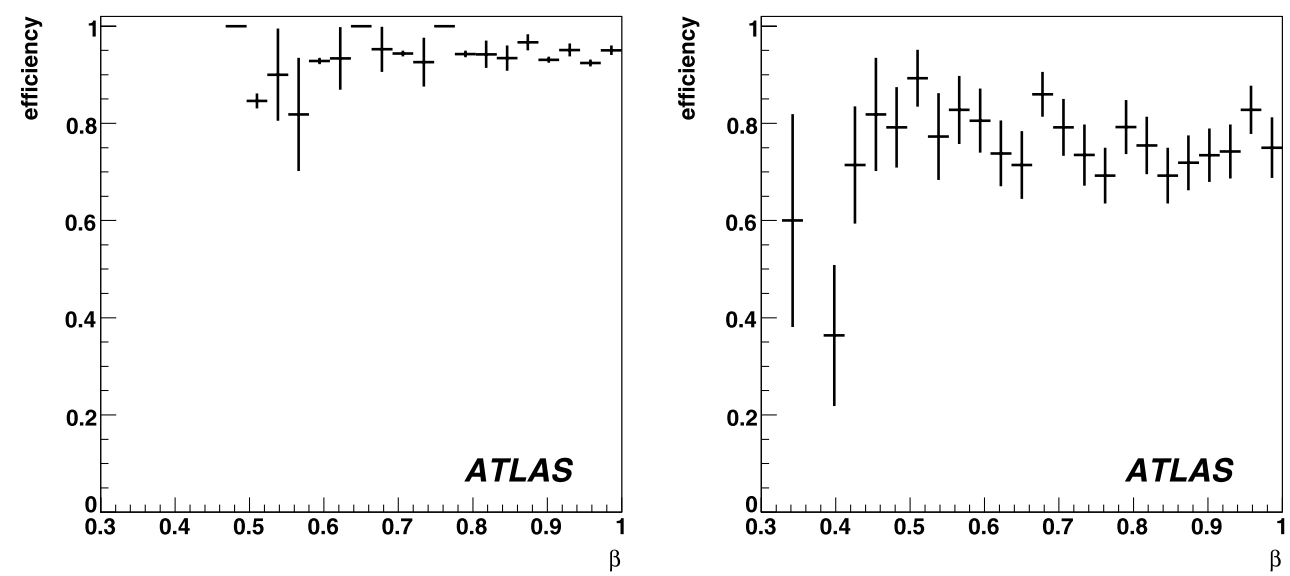

Fig. 13 Reconstructed mass of GMSB5 sleptons generated with masses of 100.2 and $102.3 \mathrm{GeV}$ (left) and R-Hadrons generated with a mass of $300 \mathrm{GeV}$ (right). The sleptons mass is centered at $102 \mathrm{GeV}$ with a $\sigma$ of $16 \mathrm{GeV}$, and the R-Hadrons mass is centered at $301 \mathrm{GeV}$ with a $\sigma$ of $64 \mathrm{GeV}$

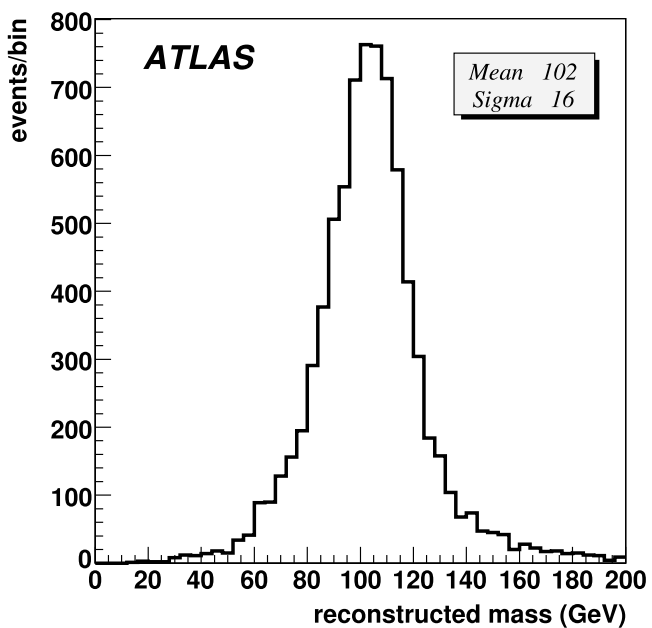

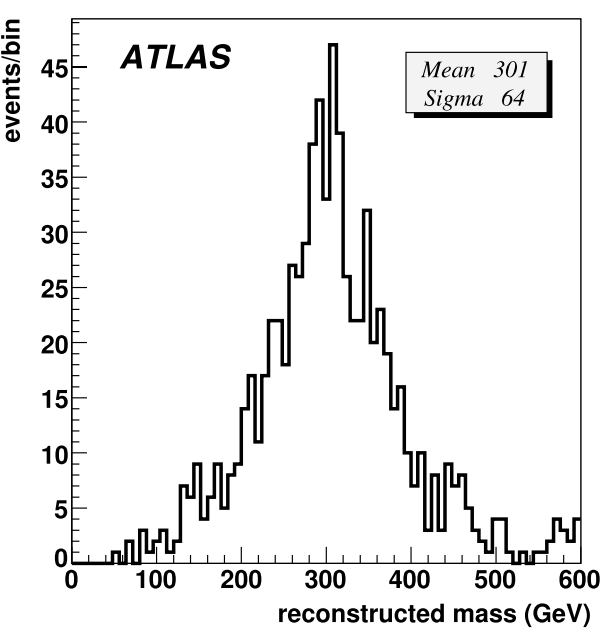

Hadrons with a mass of $300 \mathrm{GeV}$ (right). The resolutions are nearly the same for sleptons of different types and sources. The R-Hadrons are expected to lose more energy in the calorimeter, and have a wider distribution of energy loss, which could explain the worse resolution. Figure 12 shows the efficiency to reconstruct a $\beta$ within \pm 0.1 from the true value for sleptons (left) and R-Hadrons (right). Figures 11 and 12 include all slow particles with a track in the inner detector whether or not they were reconstructed as muons.

Figure 13 shows the reconstructed mass for GMSB5 sleptons generated with masses of 100.2 and $102.3 \mathrm{GeV}$ (left) and R-Hadrons generated with a mass of $300 \mathrm{GeV}$ (right). The sleptons mass is centered at $102 \mathrm{GeV}$ with a $\sigma$ of 16 $\mathrm{GeV}$, and the R-Hadrons mass is centered at $301 \mathrm{GeV}$ with a $\sigma$ of $64 \mathrm{GeV}$. In both cases the reconstructed mass is unbiased but wide. The mass resolution will be improved when the full track is re-fit with $\beta$ added as a fit parameter. However, the efficiency of keeping the candidates depends on slepton hits found in the segment finding stage.

The measured velocity of slow particle candidates is included in the resulting analysis object data. Thus these candidates are tagged for discovery or further analysis. Given the large data volume expected to be produced by ATLAS, without this tagging it would be almost impossible to locate events containing long lived charged particle for further study in the analysis stage

\section{Conclusions}

Heavy long lived charged particles can be discovered in ATLAS, should they exist. However, this cannot be done with the standard ATLAS tools alone. Furthermore, much of the discovery must be done before the analysis stage in the data acquisition, high level trigger and reconstruction.

We have developed a set of specific model independent tools to measure the mass of heavy, long lived, charged particles in the ATLAS trigger and reconstruction software. The tools have now become part of the ATLAS software release and will run automatically during data taking to produce a $\tilde{\tau}$ trigger at level- 2 and the event filter, and a $\beta$ estimate in the analysis object data resulting from reconstruction.

The efficiency to discover long lived charged particles can be improved by collecting data also from the beam crossing following the one in which the interaction occurred. 
This is most important for data from the muon trigger chambers, where this possibility is included in the data acquisition design.

The discovery potential of ATLAS for long lived particles is model dependent, and the ATLAS discovery reach for various models is not discussed in this paper. We tested the tools we developed on two distinct example models, GMSB point 5 and R-Hadrons with a mass of $300 \mathrm{GeV}$ in the framework of Split-SUSY. For these two models, discovery is possible at very low luminosity, given well calibrated data. However, the discovery methods we present do not depend on anything in the event other than the presence of a slow particle, and are therefore independent of the model characteristics. Discovery for a given integrated luminosity depends primarily on the production cross section of slow particles.

Acknowledgements We acknowledge the support of the Israel Science Foundation and the German-Israel Foundation for Scientific Research \& Development.

\section{References}

1. The LHC Study Group, The Large Hadron Collider accelerator project. CERN/AC/93-03(LHC), 1993

2. ATLAS Collaboration, ATLAS technical proposal. CERN/LHCC 94-43, LHCC/P2, December 1994

3. S. Tarem, S. Bressler, E. Duchovni, L. Levinson, Can ATLAS avoid missing the long lived stau? ATL-PHYS-PUB-2005-022, 2005

4. ATLAS Muon Collaboration, ATLAS muon spectrometer technical design report. CERN/LHCC 97-22, June 1997

5. M. Fairbairn et al., Phys. Rep. 438, 1-63 (2007)

6. G.F. Giudice, R. Rattazzi, Phys. Rep. 322, 419-99 (1999)

7. N. Arkani-Hamed, S. Dimopoulos, J. High Energy Phys. 06073 (2005)

8. R. Mackeprang, A. Rizzi, Eur. Phys. J. C 50, 353-362 (2007)
9. Aug. ATLAS Level-1 Trigger Group, Level-1 technical design report. ATLAS TDR 12, August 1998

10. E. Boos et al., Nucl. Instrum. Methods A 534, 250 (2004)

11. R. Mackeprang, Stable heavy hadrons in ATLAS. PhD thesis, Niels Bohr Institute, University of Copenhagen, 2007

12. D. Milstead, S. Hellmann, M. Johansen, Mass measurements of R-hadrons at ATLAS. ATL-PHYSPUB-2006-015, 2006

13. SingleParticelGun. http://www-theory.lbl.gov/ ianh/monte/ Generators/SingleParticleGun/

14. H. Baer, F.E. Paige, S.D. Protopopescu, X. Tata, ISAJET: a Monte Carlo event generator for $p p, p \bar{p}$, and $e^{+} e^{-}$interactions. hep-ph/ 0312045

15. T. Sjostrand, S. Mrenna, P. Skands, PYTHIA 6.4 physics and manual. hep-ph/0603175

16. S. Agostinelli et al., GEANT4-a simulation toolkit. Nucl. Instrum. Methods A 506, 250-303 (2003)

17. ATLAS HLT/DAQ/DCS Group, High-level trigger, data acquisition, and control technical design report. ATLAS TDR 16, November 2002

18. A. Di Mattia et al., A level-2 trigger algorithm for the identification of muons in the ATLAS muon spectrometer. ATL-DAQCONF-2005-005, 2005

19. ATLAS HLT/DAQ/DCS Group, High-level trigger, data acquisition, and control technical design report. ATLAS TDR 16, November 2002, p. 232

20. S. Hassani, L. Chevalier, E. Lancon, J.F. Laporte, R. Nicolaidou, A. Ouraou, Nucl. Instrum. Methods A 572, 77-79 (2007)

21. Th. Lagouri et al., A muon identification and combined reconstruction procedure for the ATLAS detector at the LHC at CERN, in IEEE Nuclear Science Symposium, vol. 3, 19-25 October 2003, pp. $1545-1548$

22. S. Tarem, Z. Tarem, N. Panikashvili, O. Belkind, MuGirl-Muon identification in ATLAS from the inside out, in IEEE Nuclear Science Symposium, vol. 1, May 2007, pp. 617-621

23. S. Ambrosanio, B. Mele, S. Petrarca, G. Polesello, A. Rimoldi, Measuring the SUSY breaking scale at the LHC in the slepton NLSP scenario of GMSB models. CERN-TH-2000-206, October 2000

24. G. Polesello, A. Rimoldi, Reconstruction of quasi-stable charged sleptons in the ATLAS muon spectrometer. ATL-MUON-99-006, October 99 